\title{
Multipath routing with adaptive playback scheduling for Voice over IP in Service Overlay Networks
}

\author{
Hong Li and Lorne Mason \\ Electrical and Computer Engineering Department \\ McGill University, Montreal, Canada \\ Email: viselia.li@mail.mcgill.ca, lorne.mason@mcgill.ca
}

\begin{abstract}
Voice over IP (VoIP) quality of service provision over the best effort Internet remains a challenging task since an interactive level conversation has strict delay, loss and delay jitter requirements. The ITU-T E-model [1] defines R-factor to measure the subjective quality of VoIP phone calls. Multipath routing and adaptive playback scheduling have been proposed to improve the quality of VoIP phone calls. However, it is still not clear how to select a multipath at the sender so that the best quality is achieved for voice signals. We propose a novel mechanism to select the optimal multipath that provides the best R-factor for VoIP phone calls with adaptive playback scheduling being applied at the receiver. We evaluate the VoIP quality for the proposed mechanism by comparing the R-factor of the VoIP calls sent through the optimal multipath with that of the VoIP calls sent through the direct path (the shortest hop path decided by the underlying network). The results show that the proposed method can raise the VoIP quality and provide much more stable quality for VoIP calls.
\end{abstract}

\section{INTRODUCTION}

VoIP is becoming more and more popular because of its low price. There are also numerous other benefits associated with VoIP since it is much easier to create new multi-media service combinations if every component service employs the IP protocol. Indeed this is a primary driver of service convergence long sought by network operators. When VoIP was in its infancy, users were prepared to tolerate poor quality as the service was effectively free, but as more and more voice traffic is being transported as VoIP customers now want toll quality service.

However, the current best effort Internet cannot satisfy the QoS requirement for VoIP [2]. While network level QoS via IntServ or MPLS/DiffServ remains a goal of network engineers, another approach, referred to as Service Overlay Networks (SONs), has been employed by VoIP operators like Skype [3]. Service Overlay Network has mechanisms customized for specific services. It consists of multiple overlay nodes/gateways interconnected by virtual connections over the current best effort Internet, as shown in Fig. 1. It is a cost efficient way of providing QoS mechanisms on top of the current best effort Internet.

In this paper, we proposed a novel multipath routing selection algorithm in Service Overlay Networks. The multipath routing mechanism assumes adaptive playback scheduling is used at the receiver. The selected optimal multipath provides the best quality for Voice over IP in terms of R-factor. We also propose a novel framework of communicating network

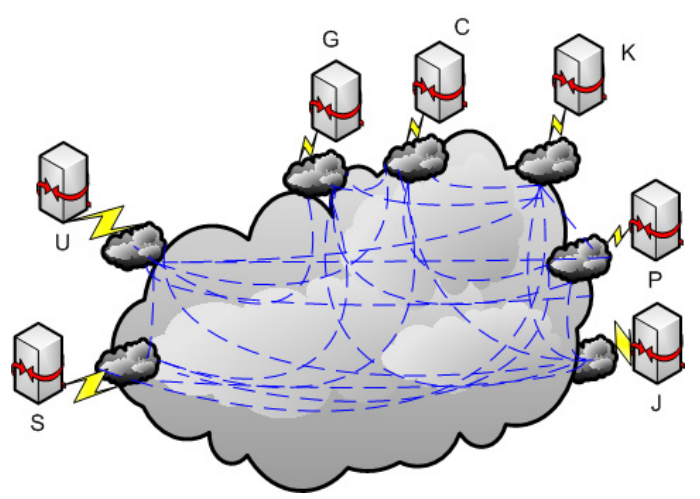

Fig. 1. Service Overlay Network. The overlay nodes are interconnected by virtual connections over the current best effort Internet.

performance characteristics where pre-processing of network measurements and data fusion center are involved. In the data fusion center optimal multipath decisions are made based on the feature vectors of the overlay link performance characteristics.

\section{RELATED WORK}

In paper [4] a VoIP specific SON architecture was proposed where source to destination path monitoring was tightly integrated with SIP [5] in order to determine the best quality path at call set up. In paper [6] an alternative approach, which used minimum delay SON routing, employed several SON nodes in tandem on an end-to-end path. VoIP packets were stored at each SON node along the path and retransmission was used to reduce packet loss.

Multipath routing in Service Overlay Networks is known to be able to substantially reduce the network losses (especially bursty losses) and delay jitters. Adaptive playback scheduling is a technique used at the receiver to adjust the playback delay adaptively to the network delay variation and to tradeoff the playback delay and playback loss for the received voice signal. Multipath selection for video streaming with multiple description coding has been proposed [13], where fixed playback scheduling was used at the receiver. However, no work has been reported on optimal multipath selection for Voice over IP that cooperates with adaptive playback scheduling.

Our approach is similar to [4] in that we investigate SONs with a relatively small number of service overlay nodes and consider only the direct and two hop paths when setting up 
a VoIP call. Our proposal differs from [4] in that we also employ path diversity which requires setting up two distinct paths for each VoIP session. Paper [9] showed the benefits of path diversity and adaptive playback scheduling for VoIP. However, they didn't show how the multipath can be selected for path diversity.

\section{PROBLEM STATEMENT}

\section{A. R-factor: a measure for VoIP quality}

R-factor defined in ITU-T E-model [1] is a score It measures the subjective voice quality under many mouth-to-ear impairments, (e.g. environment noise, echo, coding/decoding distortion, and quantization). As we are considering overlay routing and playback scheduling in SONs, only delay and loss impairments are necessary for evaluating R-factor on different paths. Then the R-factor can be written as a function of delay and loss $\mathrm{R}($ delay,loss $)=93.2-\mathrm{I}_{d}($ delay $)-\mathrm{I}_{e}($ loss $)$, where $\mathrm{I}_{d}($ delay $)$ and $\mathrm{I}_{e}$ (loss) measure the impairments caused by the total end-to-end delay and loss respectively. Here we don't include the whole formula for R-factor computation due to the space limit and the complexity of the formula. Interested audience can refer to [1] for detail. One thing to note is that the end-to-end delay includes both the network delay and the playback delay at the receiver, and similarly for the end-to-end loss.

\section{B. Problem: Optimal multipath selection for VoIP in service overlay networks}

Service overlay networks provide a cost effective way of deploying QoS mechanisms for VoIP over the best effort Internet. An overlay network can be represented as a graph $G=(V, E)$, where $V$ is the set of overlay nodes, and $E$ is the set of overlay links.

Multipath routing can be used to send redundant information on a second path and it takes advantage of the uncorrelated loss and delay characteristics of the two paths. The redundant information sent will be used by the receiver in order to recover the signal in case of packet loss. However, no results have been reported on how the optimal multipath can be selected to obtain the best R-factor for VoIP calls.

For a full mesh overlay network, it is practical to consider only overlay paths comprising of either a one-hop overlay link or two hops overlay links. We refer to the one-hop overlay paths as direct paths and the two-hop overlay paths as concatenated paths. Multipath is considered as two parallel overlay paths. In this work we assume the overlay links in a concatenated overlay path and the parallel overlay paths are all independent, which is possible since we have a maximum of two hop overlay links and two parallel overlay paths.

Let $\hat{d}$ and $\hat{l}$ represent the estimate of the total end-to-end delay $d$ and loss $l$ respectively, then the estimate of the Rfactor for a VoIP call is:

$$
\hat{\mathrm{R}}(\hat{d}, \hat{l})=93.2-\mathrm{I}_{d}(\hat{d})-\mathrm{I}_{e}(\hat{l})
$$

We denote the set of candidate overlay paths for a sourcedestination overlay node pair as $S$, which includes the direct path and all the concatenated paths. Hence, the set of possible multipath between the source-destination pair is $S \times S$.

The optimal multipath selection problem can be written as:

$$
\left(s^{*}, t^{*}\right)=\operatorname{argmax}_{(s, t) \in S \times S, s \neq t} \hat{\mathrm{R}}\left(\hat{d}_{[s ; t]}, \hat{l}_{[s ; t]}\right)
$$

\section{Solution: Optimal MUltipath ROUTING FOR VOIP}

In order to maximize the quality for VoIP calls, we propose a novel optimal multipath routing mechanism based on the estimated R-factor. The advantage of our proposed mechanism is that the selected optimal path not only maximizes the R-factor for VoIP calls, but also automatically guarantees a more stable quality for voice calls. The stability of the optimal multipath comes from the fact that the proposed end-to-end delay estimation method accounts for the network delay variations by cooperating with the adaptive playback scheduling at the receiver.

In order to estimate the R-factors on multipath and choose the best multipath accordingly, there are two challenges: one is the estimation of the end-to-end delay and loss on a multipath, and the other one is the communication of the network performance characteristics for optimal multipath decisions.

The details of the challenges and their solutions are presented in the following subsections.

\section{A. Estimation of the end-to-end delay and loss on a multipath}

One challenge of solving the optimization problem lies in the estimation of the end-to-end delay $\hat{d}$ on a path.

If we consider the end-to-end delay $d$ of a voice call, it should include not only the network delay $n_{d}$ which can be measured by sending active probes

The playback delay $\mathrm{p}_{d}$ at the receiver should not be set as a fixed value, because then all packets arriving later than this value will be discarded, which can lead to a large playback loss especially when delay jitter varies a lot in a dynamic network environment.

1) Adaptive playback scheduling: We consider adaptive playback scheduling [8][9] at the receiver, where the empirical network delay CCDF (Complementary Cumulative Distribution Function) $\mathrm{H}_{n_{d}}^{r}(x)$ is stored for the last $N$ voice packets received and the playback delay for the next packet is decided according to the $\mathrm{H}_{n_{d}}^{r}(x)$. With this approach, if the acceptable playback loss rate is $p_{l}$, the receiver automatically adjusts the playback delay as $\mathrm{p}_{d}=\mathrm{H}_{n_{d}}^{r(-1)}\left(p_{l}\right)-n_{d}$ given that the next packet received has a network delay of $n_{d}$. It can be seen that $\mathrm{E}\left(\mathrm{p}_{d}\right)=\mathrm{H}_{n_{d}}^{r(-1)}\left(p_{l}\right)-\mathrm{E}\left(n_{d}\right)$ if the network delay is stationary with $\mathrm{CCDF} \mathrm{H}_{n_{d}}^{r}(x)$ and the mean network delay is $\mathrm{E}\left(n_{d}\right)$.

Assuming the CCDF for the delays measured by the sender is $\mathrm{H}_{n_{d}}(x)$ and the CCDF for the network delays measured at the receiver is $\mathrm{H}_{n_{d}}^{r}(x)$, and they are the same and slowly varying, then the sender is able to estimate the playback delay $\mathrm{p}_{d}$ with $\hat{\mathrm{p}}_{d}$.

It is important to include $\hat{\mathrm{p}}_{d}$ in the end-to-end delay estimation $\hat{d}$ for the following reasons.

1) For two paths with the same mean network delay $\mathrm{E}\left(n_{d}\right)$, the path with heavier tail distribution, i.e. with larger 


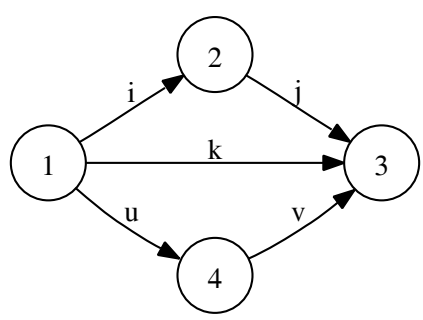

Fig. 2. Direct path and concatenated paths between node 1 and 3

$\mathrm{H}_{n_{d}}^{r(-1)}\left(p_{l}\right)$, will have a larger expected playback delay since $\mathrm{E}\left(\mathrm{p}_{d}\right)=\mathrm{H}_{n_{d}}^{r(-1)}\left(p_{l}\right)-\mathrm{E}\left(n_{d}\right)$ and thus a longer expected end-to-end delay. Therefore, the heavy tail of a network delay distribution indicates a large delay variation on a path, which should be taken into consideration when the routing decision is made.

2) The computation of R-factor requires knowing the total end-to-end delay and loss which include the playback delay and loss by definition.

Let $\hat{\mathrm{p}}_{d}\left(p_{l}\right)$ represent the estimate of the playback delay $\mathrm{p}_{d}$ at a certain tolerable playback loss $p_{l}$. Let the empirical CCDF of the network delay $n_{d}$ on a path be $\mathrm{H}_{n_{d}}(x)$, then $\hat{\mathrm{p}}_{d}\left(p_{l}\right)=$ $\mathrm{H}_{n_{d}}^{-1}\left(p_{l}\right)-n_{d}$, and the estimated total end-to-end delay $\hat{d}$ on the path is given by equation (3).

$$
\hat{d}=\mathrm{H}_{n_{d}}^{-1}\left(p_{l}\right)=n_{d}+\hat{\mathrm{p}}_{d}\left(p_{l}\right)
$$

As $\mathrm{p}_{d}\left(p_{l}\right)$ is a dynamic value set at the receiver, the sender has to estimate the adaptive playback delay $\hat{\mathrm{p}}_{d}$ by estimating the CCDF of the network delay $\mathrm{H}_{n_{d}}(x)$ on a multipath, so that the end-to-end delay $\hat{d}$ can be estimated.

However, since only network delay measurements on a single hop overlay link is available, we have to estimate the CCDF of network delays on multipath which is going to be discussed in the next few subsections.

2) Estimation of the network delay and loss on a concatenated path: In a service overlay network $\mathrm{G}=(\mathrm{V}, \mathrm{E})$, let $n_{d_{j}}$ and $n_{l_{j}}$ represent the network delay and the network loss on an overlay link $j, j \in E$,

Given the network delay distribution of each overlay link, we can find the network delay distribution on a concatenated overlay path. As shown in Fig. 2, link i represents an overlay link between overlay node 1 and 2 . For the convenience of representation we denote $[i, j]$ as the concatenated path formed by overlay link $i$ and $j, i, j \in E$. Let $\mathrm{F}_{n_{d_{i}}}(x)$ and $\mathrm{F}_{n_{d_{j}}}(x)$ represent the CDF (Cumulative Distribution Function) of the network delays on overlay link $\mathrm{i}$ and $\mathrm{j}$. Then the CCDF $\mathrm{H}_{n_{d_{[i, j]}}}(x)$ of the network delay on a concatenated path [i,j] can be computed from $\mathrm{F}_{n_{d_{i}}}(x)$ and $\mathrm{F}_{n_{d_{j}}}(x)$ by equation (5), assuming the independence of $n_{d_{i}}$ and $n_{d_{j}}$. Similarly, the network loss $n_{l_{[i, j]}}$ on the concatenated path [i,j] can also be computed from $n_{l_{i}}$ and $n_{l_{j}}$ as in equation (6).

$$
\begin{aligned}
n_{d_{[i, j]}} & =n_{d_{i}}+n_{d_{j}} \\
\mathrm{H}_{n_{d_{[i, j]}}}(x) & =\operatorname{Pr}\left\{n_{d_{[i, j]}} \geq x\right\} \\
& =1-\mathcal{F}^{-1}\left(\mathcal{F}\left(\mathrm{F}_{n_{d_{i}}}(x)\right) \cdot \mathcal{F}\left(\mathrm{F}_{n_{d_{j}}}(x)\right)\right) \\
n_{l_{[i, j]}} & =1-\left(1-n_{l_{i}}\right) \cdot\left(1-n_{l_{j}}\right)
\end{aligned}
$$

3) Estimation of the network delay and loss on a multipath: We consider multipath consisting of two parallel paths for each source-destination pair. For convenience of representation, we denote a multipath as $[\mathrm{s} ; \mathrm{t}]$ with two parallel overlay paths $\mathrm{s}$ and $t$, where $s$ and $t$ are two alternative overlay paths in the set of candidate paths between a source-destination pair. The delay on the multipath $[s ; t]$ is the minimum of the delays on path $\mathrm{s}$ and $\mathrm{t}$.

$$
\begin{aligned}
n_{d_{[s ; t]}} & =\min \left(n_{d_{s}}, n_{d_{t}}\right) \\
\mathrm{H}_{n_{d_{[s ; t]}}}(x) & =\operatorname{Pr}\left\{n_{d_{[s ; t]}} \geq x\right\} \\
& =\left(1-\mathrm{F}_{n_{d_{s}}}(x)\right) \cdot\left(1-\mathrm{F}_{n_{d_{t}}}(x)\right) \\
n_{l_{[s ; t]}} & =n_{l_{s}} \cdot n_{l_{t}}
\end{aligned}
$$

The CCDF of the delay on the multipath $[s ; \mathrm{t}]$ can be computed from equation (8).The network loss on the multipath is the product of losses on two paths as shown in equation (9). The method to estimate delay distribution and losses on two parallel paths given above is easy to extend to the case with more than two parallel paths.

4) Estimation of the end-to-end delay and loss on a multipath: Using equation (4)-(8), the estimated end-to end delay $\hat{d}_{[s ; t]}$ on the multipath $[s ; t]$ can be estimated according to equation (3), as shown in equation (10), where $p_{l}$ is the preset tolerable playback loss rate at the receiver, e.g. $p_{l}=0.01$. The end-to-end loss on the multipath $[s ; t]$ is the sum of the network loss and the playback loss as given in equation (11).

$$
\begin{gathered}
\hat{d}_{[s ; t]}=\mathrm{H}_{n_{d_{[s ; t]}}^{-1}}\left(p_{l}\right) \\
\hat{l}_{[s ; t]}=n_{l_{s}} \cdot n_{l_{t}}+\left(1-n_{l_{s}} \cdot n_{l_{t}}\right) * p_{l}
\end{gathered}
$$

B. Communication of the network performance information for optimal multipath selection

In service overlay networks, each overlay node only measures the network delays and losses from itself to all other overlay nodes. Therefore, in order to estimate the end-to-end delays and losses on the concatenated overlay paths, the delay distributions on other overlay links have to be communicated efficiently. However, the communication cost would be high when delay distributions are non-parametric.

We propose a scheme whereby only feature vectors of the network measurements are communicated and they are sent to a data fusion center, as illustrated in Fig. 3, where R-factor on multipath are estimated and optimal multipath are selected. The optimal multipath selection algorithm is given in table I.

1) Feature vector for the network performance characteristics: We have given the formula for computing the network delay distribution on concatenated paths in equation (8). For a non-parametric representation of the distributions $\mathrm{F}_{n_{d_{i}}}(x)$ and $\mathrm{F}_{n_{d_{j}}}(x)$, it is difficult to evaluate $\mathrm{H}_{n_{d_{[i, j]}}}(x)$ from the convolution of $\mathrm{F}_{n_{d_{i}}}(x)$ and $\mathrm{F}_{n_{d_{j}}}(x)$, and thus it is hard to evaluate the delay distribution on concatenated paths and on multipath.

Research on network delay measurements found that the delay distributions are slowly varying and the network delay 


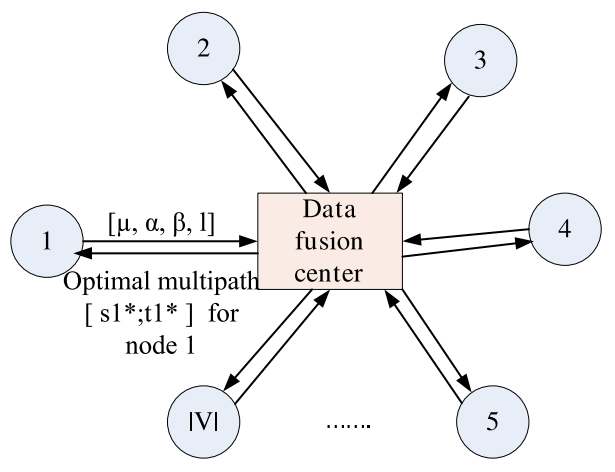

Fig. 3. Overlay nodes collect and pre-process network performance measurements, then the pre-processed network performance feature vectors $[\mu, \alpha, \beta, l]$ are sent to data fusion center to make optimal multipath routing decisions.

TABLE I

OPTIMAL MULTIPATH SELECTION ALGORITHM

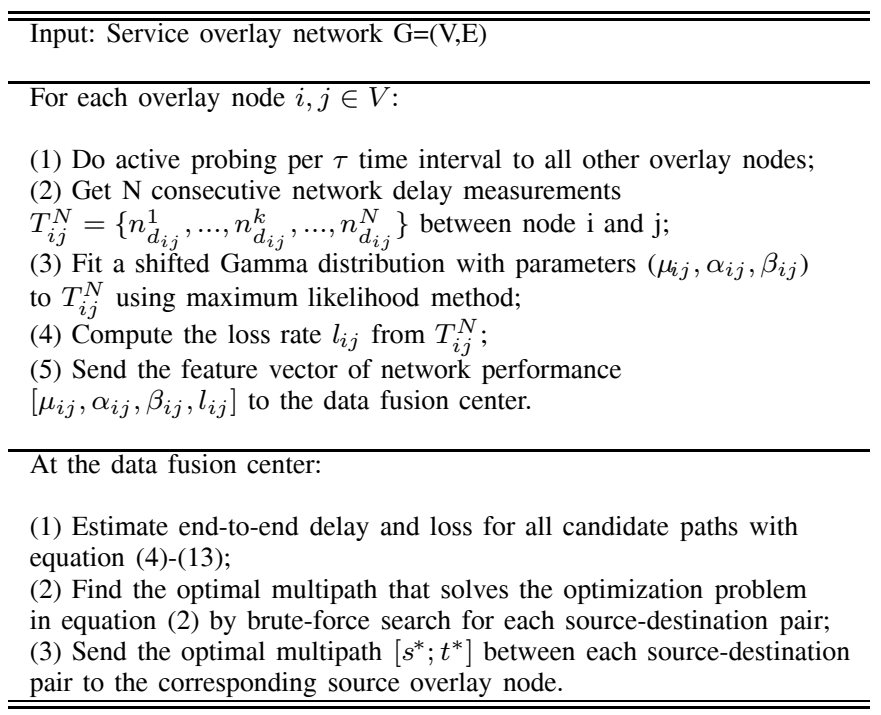

distributions on different links at different times vary in shape and can be fitted to a shifted Gamma distribution [10]. The PDF (Probability Density Function) of a shifted Gamma distribution is given in equation (12), where $\mu>0$ is the shift parameter, $\alpha>0$ is the shape parameter, $\beta>0$ is the scale parameter.

$$
f(x)=\frac{\left(\frac{x-\mu}{\alpha}\right)^{(\beta-1)} \cdot \exp \left(-\frac{x-\mu}{\alpha}\right)}{\alpha \cdot \Gamma(\beta)}
$$

The total propagation delay in an overlay link is represented by the constant value $\mu$. We use the Gamma distribution, i.e. $\mu$ is set to 0 , to model the total queuing delay in an overlay link. The shifted Gamma distribution is fitted to the real delay measurements collected by a network operator in Asia [7] and also fitted to the synthesized delay traces derived for the fractional Brownian motion traffic based on Norros' paper [11].We found that the Gamma distribution gives a good fit to the queuing delays based on Chi-square test (Weibull distribution also fits well), as shown in Fig. 4.

Therefore, the parameters of a shifted gamma distribution can be used to represent the distribution of the network delay

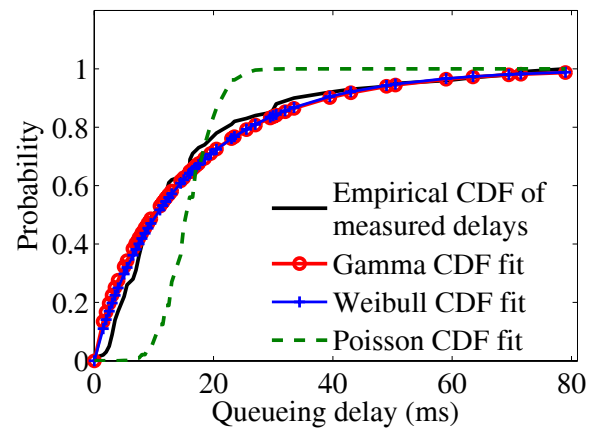

Fig. 4. Distribution fitting to network queuing delay samples (the propagation delay has been removed from the measured network delay samples)

on an overlay link, i.e., the feature vector for the network delay and loss $l$ on an overlay link $i$ can be written as $\left[\mu_{i}, \alpha_{i}, \beta_{i}, l_{i}\right]$.

2) Optimal multipath selection in data fusion center: Once the data fusion center receives the feature vectors of network performance on all the overlay links, the parameters of the delay distributions on all concatenated paths can be approximated. For the shifted Gamma distributed network delay $n_{d_{i}}$ and $n_{d_{j}}$ with parameters $\left(\mu_{i}, \alpha_{i}, \beta_{i}\right)$ and $\left(\mu_{j}, \alpha_{j}, \beta_{j}\right)$ respectively, the paper [12] mentioned that the distribution of the sum of two Gamma distributed random variables can be approximated as a Gamma distribution.Therefore, we can use shifted gamma distribution to approximate the distribution for the network delay $n_{d_{[i, j]}}$ on the concatenated path [i,j] with parameters $(\mu, \alpha, \beta)$ as given in equation (13) [12].

$$
\begin{aligned}
\mu & =\mu_{i}+\mu_{j} \\
\alpha & =\alpha_{i}+\alpha_{j} \\
\beta & =\frac{\alpha_{i} \cdot \beta_{i}+\alpha_{j} \cdot \beta_{j}}{\alpha_{i}+\alpha_{j}}
\end{aligned}
$$

Then the end-to-end delay and loss can be estimated for each possible multipath between a source-destination pair from equation (4)-(11). The R-factor can then be evaluated according to equation (1). Hence the optimization problem given in equation (2) can be solved by brute-force search where the entire set of possible multipath is searched and compared.The selected optimal multipath will then be sent to the corresponding source nodes.

\section{Simulation}

The proposed novel optimal multipath routing scheme given in section IV is simulated in the full mesh Service Overlay Network shown in Fig. 1. At each overlay node, active probing UDP packets are sent to all other overlay nodes to measure network delay, loss and jitter characteristics of the overlay links. Feature vectors are computed from the measured delay and loss characteristics for all overlay links, and then sent to the data fusion center. The communication overhead between the overlay nodes and the data fusion center is kept small by only transmitting the feature vectors. The data fusion center computes the optimal multipath for all source-destination pairs. 


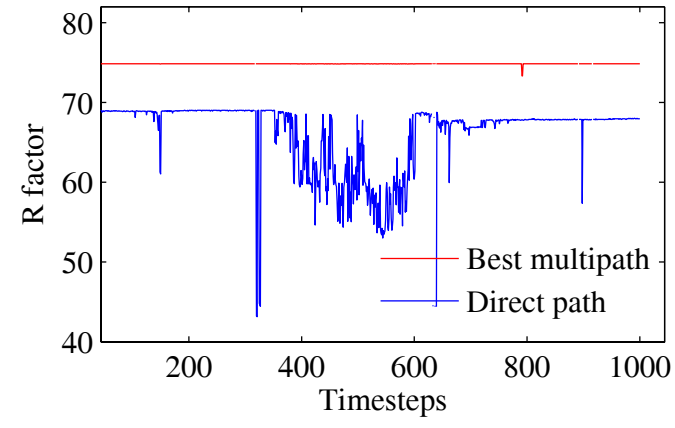

Fig. 5. R-factor on the optimal multipath (upper line) VS R-factor on direct path for a source-destination pair.

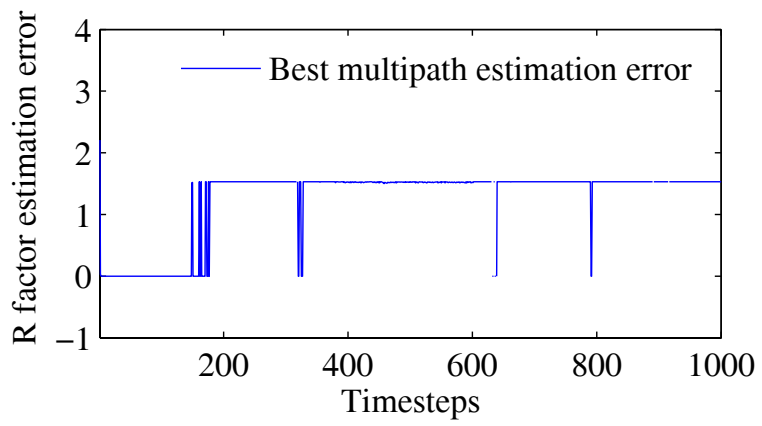

Fig. 6. Difference between the R-factor on the real optimal multipath and that on the estimated optimal multipath. The real optimal multipath is evaluated at the receiver based on the R-factor received by voice traffic on all possible multipaths. The estimated optimal multipath is evaluated at the sender based on the estimated R-factor for all possible multipaths.

The quality of the selected optimal multipath is evaluated by R-factor. VoIP calls are encoded in G.729A with VAD. At the receiver of the voice call adaptive playback scheduling is applied. We evaluate the R-factor for the voice calls sent through the estimated optimal multipath.The real optimal multipath is the multipath that gives the best R-factor when voice quality is evaluated at the receiver.

Fig. 5 shows an example where the optimal multipath increases the R-factor by a minimum of 5 and up to 32 when the direct overlay path is in bad condition, and the R-factor on the optimal multipath is much more stable compared to that for the direct path. Fig. 6 shows the difference between the Rfactor on the real optimal multipath and that on the estimated optimal multipath. The difference is due to the approximation of the network delay distribution on the concatenated paths. As you can see, the difference is very small, which means the estimated optimal multipath, which is selected based on the approximated shifted Gamma distributions, can provide voice quality close to that given by the real optimal multipath. Simulation results on other source-destination pairs show similar results as well.

\section{CONCLUSION}

In this paper, we explore the potential use of shifted Gamma distribution to estimate the end-to-end network delay distri- bution. We propose an optimal R-factor multipath selection scheme that cooperates with adaptive playback scheduling scheme at the receiver to provide optimal quality for voice calls.

The simulation shows that the proposed optimal multipath selection algorithm gives much more stable R-factor than that on the direct paths, and it also shows that the proposed method can improve the R-factor as shown in our example. The shifted gamma distribution approximation for the network delay distributions on concatenated paths only results in small error, as shown in Fig. 6, in choosing optimal multipath. Therefore, the estimated optimal multipath can provide as satisfactory quality for VoIP calls as the real optimal multipath can do.

\section{ACKNOWLEDGMENT}

The authors would like to thank NSERC strategic project grant SP 208010 for supporting this research. The authors would also like to thank Mahammed Ghanassi and Peter Kabal for their discussion.

\section{REFERENCES}

[1] ITU-T Recommendation G.107, "The E-Model, a computational model for use in transmission planning," March 2003.

[2] F. A. Tobagi, "Voice over IP: The Challenges Behind the Vision," Asilomar Conf. Signals, Systems, Computers, Pacific Grove, CA, pp. 410 414, Nov. 2004.

[3] S. A. Baset and H. Schulzrinne, "An Analysis of the Skype Peer-to-Peer Internet Telephony Protocol”, Technical Report CUCS-039-04, Columbia University, 2004.

[4] V. Hilt, A. Hari, M. Hofmann, "An Efficient and Robust Overlay Routing Scheme for VoIP,” Proc. ICICS 2005, Bangkok, Thailand, pp. 508-512, Dec. 2005.

[5] IETF RFC3261, "SIP: Session Initiation Protocol”, June 2002.

[6] Y. Amir, C. Danilov, S. Goose, D. Hedqvist and A. Terzin, "An Overlay Architecture for High-Quality VoIP streams," IEEE Trans. on Multimedia, Vol. 8, No. 6, Dec. 2006.

[7] H. Li and L. Mason, "Synthesis of network delays for voice packets in Service Overlay Networks", Qshine'07, Vancouver, Canada, August, 2007.

[8] Y. J. Liang, E. G. Steinbach, and B. Girod, "Real-Time Voice Communication over the Internet Using Packet Path Diversity," Proc. ACM Int Conf. Multimedia, vol. 9, pp. 431-440, Sept. 2001.

[9] M. Ghanassi and P. Kabal, "Optimizing Voice-over-IP Speech Quality Using Path Diversity," IEEE Int. Workshop Multimedia Signal Processing, Victoria, BC, pp. 155-160, Oct. 2006.

[10] A. Mukherjee, "On the Dynamics and Significance of Low Frequency Components of Internet Load," Internetworking: Research and Experience 5, No. 4, pp. 163-205, 1994.

[11] I. Norros, "On the Use of Fractional Brownian Motion in the Theory of Connectionless Networks," IEEE Journal on selected areas in communications, Vol. 13, No. 6, August 1995.

[12] H. C. S. Thom, "Approximate convolution of the Gamma and mixed Gamma distributions,"Monthly weather review, Vol. 96, No. 12, pp. 883886, Dec. 1968

[13] A. Begen and Y. Altunbasak and O. Ergun and M. H. Ammar, "Multipath selection for multiple description video streaming over overlay networks", Signal Processing: Image Communication, Vol. 20, Iss. 1, Jan. 2005, pp. 39-60. 